\title{
Tinnitus and lts Effect on Working Memory and Attention
}

\section{Susan Rossiter Catherine Stevens Gary Walker}

MARCS Auditory Laboratories, University of Western Sydney, South Penrith, New South Wales, Australia
Purpose: In 2 experiments, the assumption that continual orienting to tinnitus uses cognitive resources was investigated. It was hypothesized that differences in performance of tinnitus and control groups would manifest during demanding or unfamiliar tasks that required strategic, controlled processing and that reduced performance was not related solely to levels of anxiety.

Method: Nineteen participants with chronic, moderate tinnitus—-matched with a control group for age, education, and verbal IQ-completed auditory verbal working-memory and visual divided-attention tasks, with task order counterbalanced across participants. Results: As hypothesized, reading span of the tinnitus group was significantly shorter than that of the control group (Task 1). In Task 2, the tinnitus group recorded slower reaction times and poorer accuracy in the most demanding dual task context. Covariate analyses revealed that differences in task performance were not attributable to anxiety scale scores.

Conclusions: Complaints of the distracting effects of tinnitus have a basis in performance test outcomes. Future research should investigate effects of severe tinnitus and possible effects of hearing loss. At the level of theory development, results from this study suggest that tinnitus affects cognition to the extent that it reduces cognitive capacity needed to perform tasks that require voluntary, conscious, effortful, and strategic control.

KEY WORDS: tinnitus, working memory, divided attention, anxiety, controlled processes, automatic processes

$T$ innitus, the perception of sound in the absence of corresponding auditory stimulation, is a widespread phenomenon. Estimates of the incidence of tinnitus in the general population vary widely. Epidemiological studies, in which an attempt is made to include every member of the designated population in the sample, are unique in that they can provide accurate estimates of the prevalence of tinnitus, free from the biasing effects of drawing samples from clinical populations or calling for volunteers. A recent epidemiological survey conducted on the aged population (55 years and over) of a township west of Sydney, Australia, revealed that $30 \%$ of this group had experienced at least one episode of tinnitus in the year preceding the interview. This study was composed of $26 \%$ of participants with normal threshold acuity and $35 \%$ of participants with a hearing loss (Sindhusake et al., 2003). Sindhusake et al. reviewed six recent population-based studies of tinnitus prevalence in the general population and arrived at a range of $13 \%$ to $18 \%$.

Chronic tinnitus can be accompanied by depression (Folmer, Griest, \& Martin, 2001; Holgers, Erlandsson, \& Barrenas, 2000), anxiety (G. Andersson \& Vretblad, 2000; Folmer et al., 2001), insomnia (Folmer et al., 2001), problems with auditory perception (Hallam, Jakes, \& Hinchcliffe, 1988; Tyler \& Baker, 1983), and poor general and mental 
health. In extreme cases, intractable tinnitus may lead to suicide (Johnston \& Walker, 1996). Fortunately, the great majority of people who have tinnitus are not significantly bothered by it. Most individuals habituate to the sound and are barely aware of its presence. Seriously debilitating tinnitus, which gives rise to the problems outlined above, is rare, accounting for something less than $3 \%$ of the general population. This, however, still represents a large number of individuals, and research aimed at understanding tinnitus and developing successful treatments and coping strategies is warranted.

\section{Tinnitus and Cognitive Function}

This article focuses on the relationship between tinnitus and cognition. It has been frequently noted that tinnitus is associated with reduced cognitive function (Gatehouse, 1991; Jacobson et al., 1996; Wilson, Henry, Bowen, \& Haralambous, 1991), and some experimental research has appeared in the last 5 years that has confirmed effects of tinnitus on aspects of selective or divided attention and working or long-term memory (e.g., G. Andersson, Erickson, Lundh, \& Lyttkens, 2000; G. Andersson, Ingerholt, \& Jansson, 2003; G. Andersson, Khakpoor, \& Lytkkens, 2002; Hallam, McKenna, \& Shurlock, 2004). There is a need for the application of objective experimental methods, wherein tinnitus is manipulated as the independent variable, to assess the relationship between self-reported tinnitus and performance on cognitive tasks (the dependent variables). Such an approach would elucidate key variables and their interaction and help identify an explanatory mechanism.

McKenna, Hallam, and Shurlock (1996) administered a battery of tests of cognitive function to people with tinnitus and those without tinnitus to examine the relationship between tinnitus and cognition. They reported that participants with tinnitus performed more poorly than a control group on arithmetic, letter cancelation (a test of sustained attention and vigilance), verbal fluency, and trail making (a test of visual conceptualization and visuo-motor tracking) tasks. This pattern held even when the effects of trait anxiety and IQ were controlled. The results suggest that tinnitus affects performance on tasks requiring memory and/or attention. G. Andersson et al. (2000) showed that selective attention, as measured by the Stroop color and word test (Stroop, 1935), was impaired among people with tinnitus; in addition, as reported by Hallam et al. (2004), performance of a tinnitus group was slower than that of a nonclinical group on a dual task. The control of attentional processes underpins these phenomena and warrants closer investigation.

Specification of physiological and anatomical correlates of tinnitus has also been sought. For example,
Walpurger, Hebing-Lennartz, Denecke, and Pietrowsky (2003) used electrophysiological techniques and demonstrated a diminished event-related potential (ERP) response to auditory stimuli among "tinnitus complainers" (p. 57). Similarly, Jacobson et al. (1996) proposed a possible electrophysiological difference in early selective auditory attention in people with tinnitus. A significant relationship between elevated cortisol levels in people with high tinnitus distress and an intolerance of that group to external auditory stimuli has been reported by Hébert, Paiement, and Lupien (2004). Their data supported the hypothesis that there is a collapse in loudness tolerance in people severely distressed by tinnitus.

Behavioral research on tinnitus and cognition encompasses the following areas: features of the experience of people with tinnitus, personality factors that play a role, and features of the environment to which people with tinnitus show differential sensitivity. It is pertinent to ask whether habituation deficit and/or loudness intolerance affects cognitive processes and, if so, what mechanism causes the effect.

In the present study, we used two experimental tasks to scrutinize the tinnitus-cognition mechanism. One experimental task involved auditory working memory (reading span) and the other task involved visual divided attention. Participants were matched for verbal IQ so that differences in reading span should have reflected an influence of tinnitus rather than verbal or general ability. The study builds from the fundamental assumption that attention to tinnitus or thought related to tinnitus uses cognitive resources. This is in keeping with the habituation-deficit concept that people with chronic tinnitus are continually orienting to tinnitus and are in a state of arousal. In cognitive terms, this means that the capacity of working memory is reduced and attention is (already) divided across at least two tasks (where one task is orienting to tinnitus). The behavioral indexes of this cognitive process should encompass reduced working-memory span, slower reaction, and a greater number of errors in difficult, divided-attention or dual task situations. In this set of initial experiments, a working-memory task involved auditory processing and the dual task involved visual and lexical processing.

The amount of attention allocated to a task is influenced by how practiced it is (Schneider \& Shiffrin, 1977; Shiffrin \& Schneider, 1977). A distinction is drawn between tasks that involve automatic processes and those that require conscious control. The former tasks are unintentional, unconscious, and do not interfere with other cognitive processes (Posner \& Snyder, 1975). Controlled processes, on the other hand, are under conscious control and demand high levels of concentration. Many tasks evolve from being controlled to being automatic as a result of practice (LaBerge, 1975, 1990). 
Examples of this include knitting, touch typing, reading Morse code, detecting objects on radar screens, and detecting objects by sonar.

The difficulty level of tests of attention can be raised by the introduction of a divided-attention task involving two concurrent tasks (e.g., reaction time to a visual stimulus plus a verbal task). Performance on one task can be monitored while the difficulty of the other is manipulated (e.g., Johnston \& Heinz, 1978; Neisser \& Becklen, 1975). In the present study, the simple divided-attention task involved reading words and the more demanding task required naming the category to which the word belonged. While performing one of these tasks, the participant would click the computer mouse button when a gray rectangle appeared in the center of the computer screen. Reaction time was recorded as the main dependent variable (Experimental Task 2).

If individuals are distracted by their tinnitus, it is seen most clearly in their performance of complex or unfamiliar tasks under conscious control. If tinnitus affects controlled but not automatic processes, people with tinnitus will perform poorly on difficult dual task tests but not on low-demand tests. On the other hand, if tinnitus has a general impedimentary effect on attention, people with tinnitus should perform poorly, relative to matched control participants, on all tasks that require attention, regardless of difficulty.

\section{The Effects of Hearing Loss and Psychological Variables}

It is plausible that peripheral auditory damage has subtle effects on the ability to extract information from complex stimulation. For example, Granick, Kleban, and Weiss (1976) reported correlations between hearing loss and performance on verbal cognitive tests in the Wechsler Adult Intelligence Scale (WAIS; Wechsler, 1955), and Schneider, Daneman, and Pichora-Fuller (2002) noted that age-related changes in speech understanding are consequences of auditory declines. Audiometric data were not available for the sample used in the present experiments. However, we established that all participants were able to hear normal conversation. Stimuli were presented aurally in Experiment 1, and experimental trials were preceded by practice trials. U. Andersson (2002) demonstrated that specific aspects of the phonological system deteriorate as a function of auditory deprivation. However, Andersson's experimental results suggest that phonological processing in verbal working memory of people with acquired hearing loss is preserved. In Experiment 1, we investigated whether moderate, chronic tinnitus affected reading span while treating anxiety scores as a covariate. Because participants were matched for verbal IQ and anxiety scores used as covariates, differences in reading span would imply a re- lationship between tinnitus and reduced verbal working memory. Experiment 2 stimuli were visual (i.e., a gray rectangle appearing at random intervals; written words).

The issue of causality is a difficult one. Does tinnitus cause a drop in performance directly, or is there a third factor involved? For example, it is well established that there is a relationship between anxiety and tinnitus (G. Andersson \& Vretblad, 2000; Halford \& Anderson, 1991; McKenna, Hallam, \& Hinchcliffe, 1991; McKenna et al., 1996; Rutter \& Stein, 1999). There is also a wellestablished relationship between anxiety and cognitive task performance (Broadbent, Broadbent, \& Jones, 1986; Eysenck \& Calvo, 1992). Could it be that the reason for the cognitive impairment is not tinnitus, but the cooccurrence of high levels of anxiety? On the other hand, tinnitus may cause the anxiety and general emotional distress that, in turn, disrupt cognitive processes. Constantly attending to the sound may increase its intrusiveness (Hallam et al., 1988). It has also been suggested that the emotional distress associated with tinnitus is heightened by its uncontrollability (Halford \& Anderson, 1991; Jakes, Hallam, Chambers, \& Hinchcliffe, 1985): Individuals become caught in a cycle of increasing negativity and anxiety and decreasing cognitive functioning (Hallam, Rachman, \& Hinchcliffe, 1984; Jastreboff \& Hazell, 1993). Newman, Wharton, and Jacobson (1997) showed that some people with tinnitus could be categorized as low self-attenders or high self-attenders. They found that the latter group was, on average, more depressed, had greater emotional distress because of tinnitus, and had greater perceived tinnitus handicap. In the present study, we used State-Trait Anxiety Inventory (STAI; Spielberger, Gorsuch, Lushene, Vagg, \& Jacobs, 1983) scores as covariates to examine the contribution of selfreported anxiety toward cognitive task performance. Tinnitus severity was measured using the Tinnitus Reaction Questionnaire (TRQ; Wilson et al., 1991).

\section{Purpose}

The general purpose of this study was to investigate the relationship between tinnitus and cognition using the experimental method. The long-term goal was to contribute to understanding the way in which chronic tinnitus disrupts cognitive performance and to use this knowledge to develop management strategies to minimize this disruption.

The specific purpose of this study was to examine the performance of individuals with moderate tinnitus and a matched control group on two established tests of cognitive function: the listening version of the Reading Span Test (Daneman \& Carpenter, 1980) and a dual task divided-attention test involving visual stimuli. The underlying cognitive processes assessed were auditory working memory (Task 1) and visual attention (Task 2). 
In some ways, the work reported here was a single experiment with the above-listed goals. The tests were carried out on the same day using the same participants. However, the experiment could also be regarded as two experiments that deal with different aspects of the problem. This fact is reflected in the structure of the article. First, we present matters common to both tasks, such as the characteristics of the sample, followed by descriptions of each test and discussion of outcomes. Last, we discuss the overall outcome of the study and show possible implications for tinnitus management.

\section{General Method Participants}

Experimental group. The experimental group consisted of 19 participants (16 men, 3 women) age 34 to 63 years $(M=48.9$ years, $S D=8.2)$. All participants were from an English-speaking background. They all reported experiencing constant, bilateral $(n=14)$ or unilateral $(n=5)$ tinnitus for more than 3 months. Ten participants had experienced tinnitus for more than 5 years, and the minimum duration was 9 months. One participant had been diagnosed with hearing loss and wore a hearing aid, and 14 others in the tinnitus group had self-reported mild to moderate hearing loss. The participants were mainly recruited through an advertisement placed in the newsletter of the Australian Tinnitus Association. A few participants learned of the study through other participants or were known to the experimenter.

This sample was not representative of the whole tinnitus population, nor was it meant to be. Therefore, the results of the research cannot be generalized to the whole population. Tinnitus need not be treated as a single phenomenon with uniform characteristics. A large percentage of tinnitus sufferers have mild tinnitus. Mild tinnitus usually undergoes habituation and may go unnoticed for long periods of time. It typically does not disrupt life or have much of an impact on anything. A representative sample of tinnitus sufferers would have to include the appropriate percentage of such cases. These contribute only "noise" to the data. To eradicate this noise and reveal the interesting results, one must test a very large sample. By deliberately recruiting people with constant, moderate to severe tinnitus, we maximized the chances of obtaining significant results from a sample of manageable size.

We did not measure participants' hearing threshold levels or try to match the experimental and control groups on this variable. This may seem unusual, given that tinnitus is an auditory phenomenon. However, in the second experimental task, all of the stimuli were presented visually. All that was required was that the participants could see the stimulus on the computer screen and could read (groups were matched on reading ability). Only in the Reading Span Test was the stimulus presented orally. The participant and experimenter were seated facing one another and about $1 \mathrm{~m}$ apart in a quiet test room. The sentences were delivered by unmonitored live voice at a normal conversational level and pace, and, during practice trials, the experimenter checked with participants that they could hear the stimulus items. Our approach to this type of experiment was to choose individuals with continuous, moderate to severe tinnitus and normal auditory thresholds or mild to moderate hearing loss. By not using individuals with moderate to severe hearing loss or worse, we hoped to avoid the problems associated with the reduced dynamic range of the ear and its associated loudness tolerance (loudness recruitment) problems (Baskill \& Coles, 1999) and the decreased frequency and temporal resolution abilities that cause signal distortion and reduced speech intelligibility. Researchers have shown that hearing loss may be associated with tinnitus and may lead to problems in conjunction with high cognitive load and/or be associated with cognitive changes (Granick et al., 1976; Pearman, Freidman, Brooks, \& Yesavage, 2000). Because of this realization, thresholds have been measured in subsequent experimental work (e.g., Stevens, Walker, \& Gallagher, 2005).

Control group. The control group, which also contained 19 participants, was selected after the experimental group. Individuals selected for the control group were from an English-speaking background and matched to individuals from the experimental group according to age, education level, occupation, and score on the National Adult Reading Test (NART; Nelson, 1991). All were referred by word of mouth, were known to the researcher, or were friends or relatives of other participants. The NART was administered to those who were considered, on the basis of the telephone interview, to be likely candidates. On the basis of the result, the individuals were either accepted into the control group and the rest of the testing was carried out or their participation was ended. The inclusion criteria were the same as for the experimental group, except that the control group had not experienced tinnitus in the preceding 6 months. Two members of the control group had been diagnosed with hearing loss and wore a hearing aid. Eight others had self-reported mild hearing loss. The group consisted of 13 men and 6 women age 30 to 63 years $(M=48.8$ years, $S D=8.8)$.

\section{Telephone Screening}

The suitability of volunteers for inclusion in the tinnitus and control groups was assessed by telephone 
interview, during which general demographic data and a brief medical history were obtained. The inclusion criteria were as follows: The participants had had constant tinnitus for at least 3 months (tinnitus group only) without any indication of spontaneous recovery (Vesterager, 1997), their corrected vision was sufficient for them to see the visual stimuli on a computer screen, they did not suffer from any medical condition that may cause cognitive dysfunction (e.g., tumor, serious head injury, dementia), and they had not recently undergone any medical procedure and were not taking any medication that could affect cognitive functioning. The latter included drugs that may cause drowsiness, confusion or agitation.

\section{Psychological Test Materials}

A single test session of 2.5 hours began with collection of demographic data and administration of the NART. Participants then completed Experimental Tasks 1 and 2 (counterbalanced), followed by three tests in random order: the STAI (Spielberger et al., 1983), the Cognitive Failures Questionnaire (CFQ; Broadbent, Cooper, Fitzgerald, \& Parkes, 1982), and the TRQ (Wilson et al., 1991).

The NART provides a quick but valid and reliable estimate of verbal IQ (Crawford et al., 1989). The NART has split-half and test-retest reliabilities of .93 and .98, respectively. ${ }^{1}$ We used the test to match members of the tinnitus and control groups. The NART raw scores were as follows: Experimental group, $M=10.00, S D=2.31$, and control group, $M=10.68, S D=2.67$. These scores related to a predicted IQ for the experimental group of $M=118.84, S D=2.24$, and for the control group, $M=119.26, S D=1.94$.

The STAI consists of two, 20-item self-report scales. State Anxiety refers to how the respondent feels "right now," whereas Trait Anxiety refers to how the respondent "generally" feels. The two scales are correlated. The maximum score on each scale is 80 . The STAI was used to help in the interpretation of results, with anxiety scores used as covariates. The mean State Anxiety score for the tinnitus group was $37.05(S D=9.40)$ and for the control group was $30.84(S D=5.68)$. Mean Trait Anxiety score for the tinnitus group was $38.74(S D=11.84)$ and for the control group was $31.90(S D=5.08)$. These mean sores are comparable with normative data reported by Spielberger et al. (1983)—State $M=35.72, S D=10.40$, and Trait $M=35.85, S D=10.91$-and scores reported by Wilson et al. (1991) in the development of the TRQ-State $M=35.85, S D=10.91$, and Trait $M=38.73, S D=12.18$.

\footnotetext{
${ }^{1}$ Split-half reliability refers to the reliability of a test by evaluating the test's overall internal consistency and dependability as a measuring device. A single test is split into two forms and a coefficient of reliability between the two is obtained.
}

The CFQ contains 25 items designed to assess the frequency of everyday slips and errors. Participants rate statements such as "Do you fail to notice signposts on the road?" from 0 (never) to 4 (very often). The CFQ provides a measure of an individual's ability to distribute attention in stressful situations, such as those involving multiple concurrent tasks (Harris \& Wilkins, 1982; Martin \& Jones, 1983; Reason, 1988). Researchers have shown the CFQ to be reliable (Broadbent et al., 1982). The CFQ scores in this study were as follows: tinnitus group, $M=46.33, S D=18.38$, and control group, $M=41.28$, $S D=9.41$. It is surprising that the groups did not differ in their results on the CFQ. The control group in the present study had higher scores than the control group in McKenna et al.'s (1996) study. The scores in our study suggest that participants in both groups, on average, indicated that they "occasionally" experienced the cognitive slips referred to in scale items. Neither group reported a severe rate of cognitive failures, but the lack of a difference between groups made it more difficult to find statistically significant differences between the groups.

The TRQ, which was administered only to the experimental group, provides an estimate of the severity of the individual's reaction to his or her tinnitus (Wilson et al., 1991). The CFQ and TRQ provide extra information about the participants. The mean TRQ score of the experimental group was $36.39(S D=22.32$, mode $=37$, range $=0-74$ ), indicating that the experimental group had, on average, moderate tinnitus. This mean score is comparable with that of other tinnitus samples. Wilson et al., for example, reported a group mean score of 32.49 $(S D=22.71)$.

The order of experimental tasks was counterbalanced to distribute serial order effects.

\section{Experimental Task 1}

The first of the experimental tasks was the Reading Span Test of Daneman and Carpenter (1980). The title is something of a misnomer in that this version of the test involves the participant listening to, rather than reading, sentence material. The test is said to be an index of working memory.

\section{$\overline{\text { Method }}$ Stimuli}

The Reading Span Test consists of 100 unrelated sentences of between 13 and 16 words, arranged in $5 \times 2$ sentence sets, $5 \times 3$ sentence sets, and so forth, up to $5 \times 6$ sentence sets. 


\section{Procedure}

The sentences were presented "live voice" to the participants, who were given $1.5 \mathrm{~s}$ at the end of each sentence to indicate if it made sense. At the end of each set of sentences, a bell sounded and the participant was required to recall the final word of all the sentences in the set. The question as to whether each sentence made sense was irrelevant to the real purpose of the test and was included to ensure that participants did not just concentrate on the final word of each sentence (Turner \& Engle, 1989). To be judged as successfully remembered, the final words of all the sentences in the set had to be correctly recalled in the correct order. The reading span of each participant was calculated as the number recalled correctly on three out of the five sets. For example, if the participant correctly recalled the last words in all five of the two-sentence sets, he or she would move on to the three-sentence sets, and so on, up to the maximum sixsentence sets. However, if, for example, he or she could only recall all of the last words in three of the five foursentence sets, he or she would be allocated a reading span of 4.0. If the participant was successful in two of the five four-sentence sets, the span would be 3.5. To ensure that participants understood the task and that the sentences were audible, several practice trials were given prior to the test.

\section{$\overline{\text { Results }}$}

The mean reading span of the tinnitus group was $3.00(S D=0.37)$ compared with $3.61(S D=0.49)$ for the control group. An independent samples $t$ test revealed that this difference was significant at better than the $5 \%$ probability level, $t(36)=4.30, p<.05$. The raw reading span scores ranged in both groups from 2.5 to 4.0. However, as Figure 1 illustrates, the lower reading spans were prevalent in the tinnitus group, with 12 of the 19 participants $(63 \%)$ recording a span of 3.0. By contrast, 10 of the 19 participants (53\%) in the control group recorded a span of 4.0.

To examine the contribution of anxiety to the results, the individual scores on the STAI were entered into an analysis of covariance (ANCOVA), with state anxiety as a covariate. The ANCOVA retained a significant effect of group (tinnitus - nontinnitus) and revealed no effect of state anxiety on reading span, $F(1,35)=0.30, p>.05$. The effect of trait anxiety on the performance of both groups was examined in separate regression analyses. Because trait and state scores are correlated and because trait is a constant variable, only the trait anxiety scores were used in this analysis. There was no correlation between reading span and trait anxiety for either the tinnitus or the control group: $r=-.10, p>.05, F(1,17)=0.17, p>.05$, and $r=.25, p>.05, F(1,17)=1.15, p>.05$, respectively.
Figure 1. Frequency distribution of raw reading span scores for tinnitus and control groups in Experiment 1.

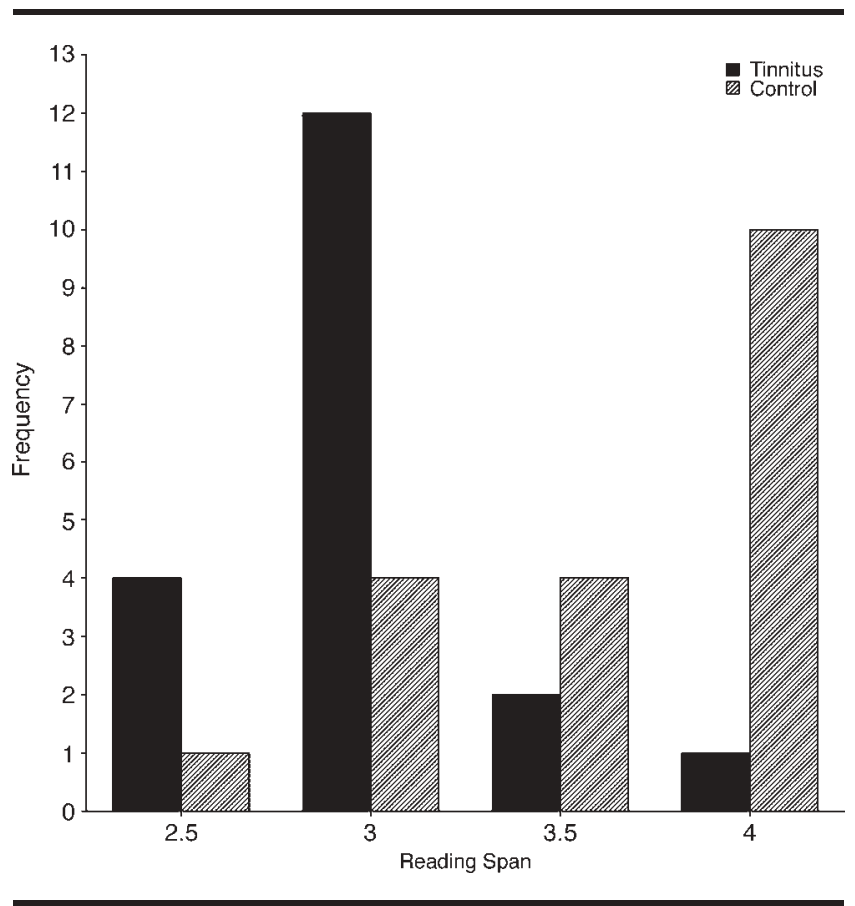

These results corroborate anecdotal accounts that some individuals with tinnitus have difficulties concentrating on the task and/or reduced capacity to store and retrieve information from working memory (Davis \& Roberts, 1996; Hallam et al., 1988). The findings are not the result of anxiety or differences in verbal IQ (as measured by the NART). The finding of a significant difference between the experimental and control groups contrasts to the lack of a difference between the two groups on the CFQ.

\section{Experimental Task 2}

Experimental Task 2 included three separate tasks involving attention and that provided conditions of increasing attentional demand. The task included a simple visual reaction-time task; a divided-attention, word-recognition task; and a divided-attention, wordcategorization task. The purpose was to investigate the ability of the two groups to divide attention between tasks in conditions of increasing difficulty.

A dual task paradigm was used, wherein the difficulty of the primary task was increased from easy to hard while reaction time to the secondary task was recorded. The $2 \times 3$ factorial design consisted of participant group (tinnitus, control) and task difficulty (baseline-single task, word recognition, category naming) conditions, with repeated measures on the latter 
factor. The dependent variables were reaction time and errors. We hypothesized that if tinnitus has an undifferentiated, general effect on cognition, performance of the tinnitus group should be poorer than that of the control group on all conditions. Alternatively, if tinnitus affects controlled but not automatic processes, the tinnitus group should perform more poorly than the control group on the category-naming, but not on the baseline or wordrecognition, conditions.

\section{$\overline{\text { Method }}$ Participants}

One member of each of the two groups failed to complete this part of the experiment.

\section{Stimuli and Equipment}

The secondary task stimulus and the sole stimulus in the baseline condition consisted of a small, gray rectangle that appeared on the computer screen and remained there until the mouse button was clicked. The rectangle appeared at random interstimulus intervals ranging from $1 \mathrm{~s}$ to $9 \mathrm{~s}$. The easy primary task consisted of 90 English words that belonged to one of three superordinate categories, namely cooking, animal, or seascape. Words were matched across superordinate categories for word length and frequency of occurrence (Baayen, Piepenbrock, \& Gulikers, 1995). A word appeared at one of the four corners of the computer screen every $1.5 \mathrm{~s}$. The task was to name the word. The same 90 words were used in the difficult primary task. The task was to name the subordinate category to which the word belonged. The experiment was programmed in TrueBasic and presented on a Macintosh laptop computer.

\section{Procedure}

The baseline task was always completed first. The order of the word-recognition and category-naming conditions was counterbalanced across participants to distribute serial order effects. As a baseline measure of reaction time, participants clicked the mouse button as quickly as possible each time a gray rectangle appeared in the center of the computer screen. After a series of practice trials, baseline data were collected across 30 trials. In the easy primary task, participants read aloud each word as it appeared in one of four corner locations on the computer screen. Concurrently, they performed the secondary task, clicking the mouse button when a rectangle appeared in the center of the screen. In the difficult primary task, rather than reading the word as it appeared on one of the corners of the screen, participants had to name the superordinate category to which the word belonged. For example, when the word "wine" appeared, they were to respond "cooking." At the same time as naming the categories aloud, participants clicked the mouse button as quickly as possible each time a rectangle appeared.

\section{$\overline{\text { Results }}$}

Mean reaction time as a function of participant group and task difficulty is shown in Figure 2. As a manipulation check, reaction time in the dual task conditions was significantly greater than in the baseline (single task) condition with participant group collapsed, $F(1,34)=$ $126.40, p<.05$. Two orthogonal, planned comparisons were used to test the hypothesis that there was no significant difference between reaction times recorded by tinnitus and control groups as they performed the low-demand, word-recognition dual task, $F(1,34)=0.28$, $p>.05$. A significant difference was found between the tinnitus and control groups on the category-naming task, $F(1,34)=4.20, p<.05$, with the tinnitus group responding significantly more slowly (Figure 2). A frequency distribution of individual participant mean reaction times in the category-naming condition is shown in Figure 3.

Errors in word reading and category naming were calculated. Although the tinnitus group made slightly more errors in the word-reading condition $(M=3.50$, $S D=2.53)$ compared with the control group $(M=2.67$, $S D=2.57)$, the results of an independent $t$ test revealed

Figure 2. Mean response time (ms) as a function of participant group and single (baseline) and dual task conditions in Experiment 2. Error bars refer to standard error of the mean.

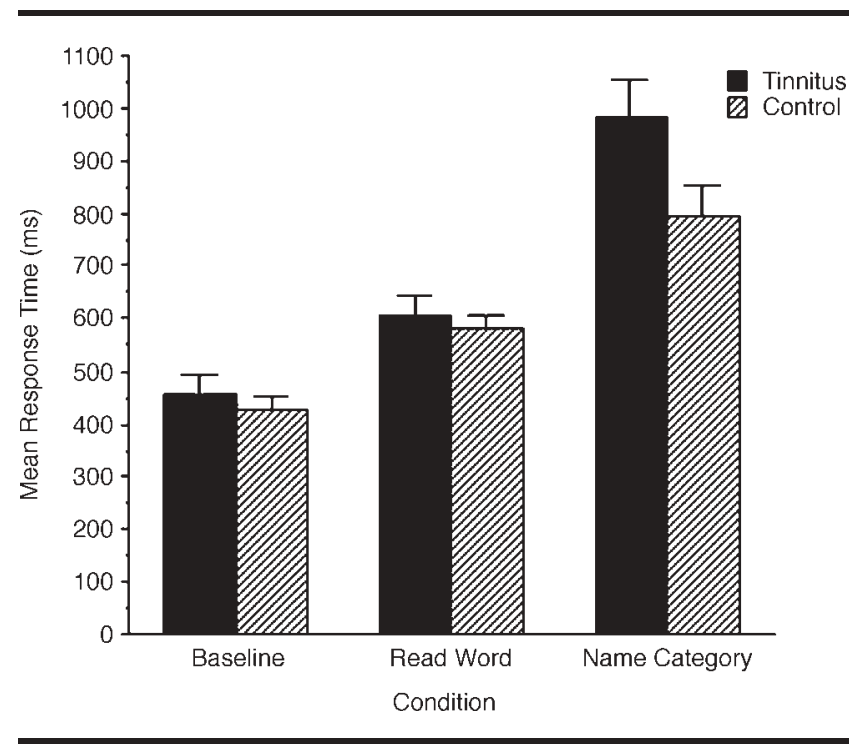


Figure 3. Frequency distribution of individual mean reaction times (ms) for tinnitus and control groups in the category naming condition in Experiment 2.

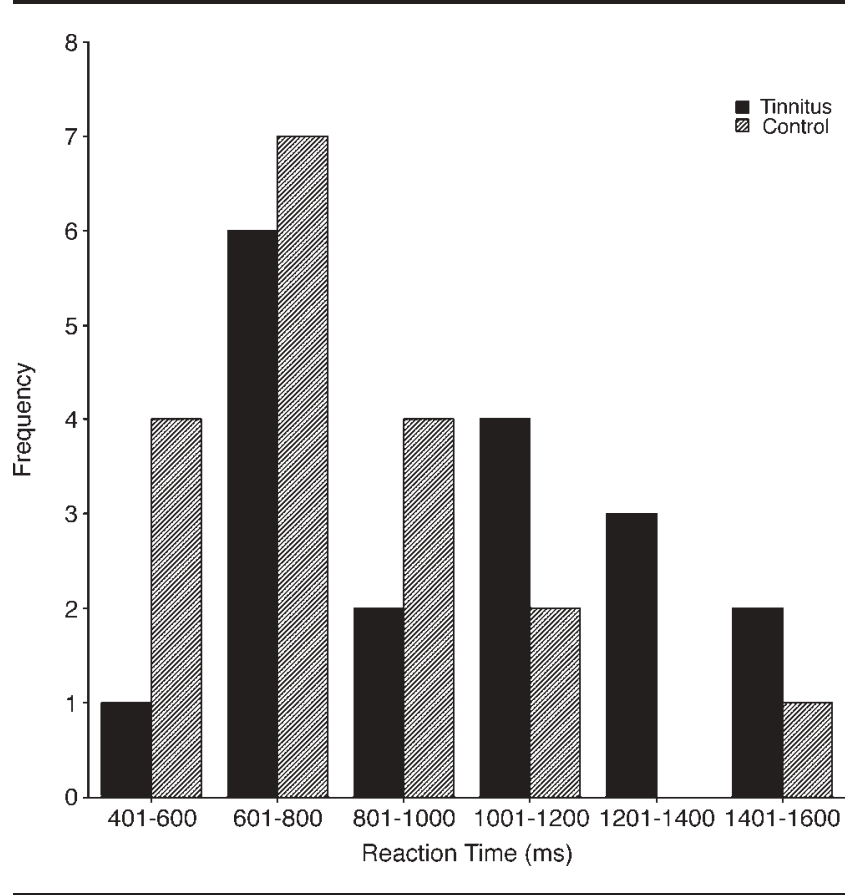

no significant difference between the groups, $t(36)=0.98$, $p>.05$. However, there was a significant difference in the number of errors recorded in the category-naming condition by the tinnitus group $(M=7.50, S D=2.72)$ compared with the control group $(M=4.28, S D=2.57)$, $t(36)=3.65, p<.05$.

An ANCOVA, with state anxiety as a covariate, revealed no significant effect of anxiety for task condition or participant group. When using trait anxiety as the covariate for the entire group, results revealed a significant main effect on the baseline, $F(1,33)=6.36$, $p<.05$, and reading conditions, $F(1,33)=6.03, p<.05$, but not the category-naming condition, $F(1,33)=3.85$, $p>.05$.

A regression analysis was conducted to investigate the effect of trait anxiety on performance in the baseline condition for tinnitus participants, separate from the control group. No significant correlations were found. A separate regression analysis was conducted for the control group but results were also not significant. Control participants' performance on the baseline and reading tasks were significantly correlated with trait anxiety: $r=-.44$, $p<.05, F(1,16)=3.75, p=.07$, and $r=-.55, p<.05$, $F(1,16)=6.99, p<.05$, respectively. No correlation was evident between trait anxiety in the control group and performance on the categorizing task: $r=-.29, p=.13$, $F(1,16)=1.41, p=.25$.

\section{$\overline{\text { Discussion }}$}

The dual task paradigm showed differences between individuals with chronic, moderate tinnitus and a control group, including some effects of anxiety on task performance. A baseline reaction-time task elicited no difference in performance of the two groups. Similarly, the lowdemand, dual task condition involving the automatic task of recognizing words revealed no significant difference. However, the more difficult category-naming task revealed significant differences in reaction times of the two groups: The mean of the tinnitus group was $150 \mathrm{~ms}$ greater than that of the control group. The principle behind the dual task paradigm is that as the primary task becomes more difficult, more attentional resources are directed to that task, with fewer resources available for the secondary task. Attentional resources are required to overcome the competing word-reading response and/or determine the superordinate category to which the word belongs (Rosch \& Mervis, 1975; Smith \& Medin, 1981).

The relationship among tinnitus, anxiety, and task performance is potentially complex. ANCOVAs revealed no effect of state anxiety on performance. Trait anxiety was related to performance of combined tinnitus and control groups on the baseline and word-reading conditions but not the category-naming condition. Participant group and anxiety had an effect, but results suggest a relationship between control participants' level of trait anxiety and performance on baseline and word-reading conditions. The slower reaction time recorded by the tinnitus group on the category-naming tasks was unrelated to state or trait anxiety scores. In future studies, depression scores could be obtained to rule out depression as contributing to poorer performance on the demanding category-naming task.

\section{General Discussion}

Two experimental tasks investigated the effect of chronic tinnitus on auditory working memory and visual divided attention. In Experimental Task 1, a significant difference in reading span was observed between the tinnitus and control groups. This indicates that working memory, over and above verbal ability (matched using the NART), is affected by chronic tinnitus. Analyses using anxiety scores as a covariate indicated that the difference in reading span of the two groups was not accounted for by differences in anxiety levels. The cognitive mechanism that underpins reduced reading span, such as capacity limitation, changing state (G. Andersson et al., 2002), or interference, requires further investigation. 
The divided-attention task used in Experimental Task 2 explored the relationship between increasingly demanding tasks and moderate, chronic tinnitus on processing speed and accuracy. The tinnitus and control groups did not differ significantly when they performed the simple baseline task. The overlearned and automatic word-recognition context in Experiment 2 also elicited no differential effects in speed or accuracy of the response. However, as hypothesized, performance of the tinnitus group was significantly poorer than that of the control group on the less familiar, category-naming, dual task condition. An additional interpretation is that tinnitus has no effect on tasks that involve involuntary, automatic processes but that it disrupts controlled, conscious cognitive processes. Such effortful processing is characteristic of the category-naming, dual task condition and the reading span task when target stimuli exceed three sentences.

Could the differences found between the experimental and control groups be the result of differences between these groups in auditory acuity and/or auditory processing ability? Experimental Task 2 involved only visual stimuli. Experimental Task 1 involved listening to speech signals, but the auditory task was an easy one, that is, to recognize meaningful sentences delivered at conversational level by a close (about $1 \mathrm{~m}$ ) speaker in a quiet test room. Participants' ability to hear speech in such conditions was checked during the practice trials prior to the test. Nonetheless, it is possible that some neural reorganization accompanies hearing loss, and this may have affected neural networks subserving memory and attention (e.g., U. Andersson \& Lyxell, 1999; Granick et al., 1976; Murphy \& Wilcox, 1999; Pearman et al., 2000; Schneider et al., 2002). It is important that the tinnitus and control group samples in this study both contained participants with some self-reported hearing loss. U. Andersson (2002) argued that although acquired hearing loss affects phonological processing, it does not affect verbal working memory. We have confidence in concluding that differences in reading span observed in Experiment 1 were the effect of tinnitus rather than mild hearing loss. In recent work (Stevens et al., 2005), hearing thresholds have been measured and participants matched as closely as possible on this variable. Indeed, it would be valuable to study a participant group that encompasses varying degrees of hearing loss and tinnitus to illuminate the contribution of these two factors.

In this study, scores on the STAI Scale were used in ANCOVAs and revealed no effect of anxiety on performance of the tinnitus group. Anxiety is not the only variable that could influence the participant's performance. It is one of many. In particular, many tinnitus sufferers report that they are depressed. Depression ranks with anxiety as the two psychological variables most likely to be involved. Therefore, the effects of depression should be examined in the same way as those of anxiety, and this will be done in future studies.

In the present study, the control and tinnitus groups, surprisingly, did not differ on the CFQ. This was a statistically useful outcome, leading to comparison of two groups of individuals reporting occasional cognitive slips: Cognitive impairment was evident only in the sample of participants who experienced moderate tinnitus. It is possible that people with tinnitus downplay effects of tinnitus on cognition, or they are unaware of subtle effects that manifest in experimental tasks conducted in controlled laboratory conditions. It should be noted too that the tinnitus group consisted of people with moderate tinnitus and that stronger effects may be observed in people with severe tinnitus and possibly higher levels of anxiety and depression.

The assumption that tinnitus affects controlled processing leads to two possible mechanisms underlying poorer cognitive task performance, namely interference with, or reduced capacity for, strategic, voluntary control. Attention resources may be disrupted or depleted due to negative thoughts, continual orienting to tinnitus (Hallam, 1986), increased self-focused and somatic attention (Newman et al., 1997), and/or the continual search for environmental sounds to mask the tinnitus. Well-designed experimental tasks are needed to distinguish interference from capacity limits as the cognitive mechanism.

Intrusiveness of tinnitus appears to decrease with time (Hallam et al., 1984), and this observation is consistent with the increasing automaticity of behavior associated with learning. It is important to note that performance of the tinnitus and control groups was comparable on the low-demand conditions. This conforms to the notion that becoming involved in activities that require some mental effort may reduce tinnitus interference and restore performance to normal (Hallam, 1986). The controlled processing hypothesis, if upheld in future investigations, has important implications for tinnitus management. If tinnitus disrupts controlled but not automatic processes, new or difficult tasks need to be practiced and rehearsed to promote transition from controlled to automatic processing. Once automatic, there should be minimal interference from any concurrent activity or stimulus, including tinnitus.

\section{Acknowledgments}

This research was conducted by the first author in partial fulfilment of the requirements for the Master of Arts degree (Honor) at the University of Western Sydney and supported by MARCS Auditory Laboratories and the School of Psychology. We thank Denis Burnham, Helen Tam, Cyril Latimer, Melinda Gallagher, and Leonid Grebennikov for their comments. 


\section{References}

Andersson, G., Erickson, J., Lundh, L., \& Lyttkens, L. (2000). Tinnitus and cognition interference: A Stroop paradigm. Journal of Speech, Language, and Hearing Research, 43, 1168-1171.

Andersson, G., Ingerholt, C., \& Jansson, M. (2003). Autobiographical memory in patients with tinnitus. Psychology \& Health, 18, 667-675.

Andersson, G., Khakpoor, A., \& Lyttkens, L. (2002). Masking of tinnitus and mental activity. Clinical Otolaryngology, 27, 270-274.

Andersson, G., \& Vretblad, P. (2000). Anxiety sensitivity in patients with chronic tinnitus. Scandinavian Journal of Behaviour Therapy, 29, 57-64.

Andersson, U. (2002). Deterioration of the phonological processing skills in adults with acquired severe hearing loss. European Journal of Cognitive Psychology, 14, $335-352$.

Andersson, U., \& Lyxell, B. (1999). Phonological deterioration in adults with an acquired severe hearing impairment: A deterioration in long-term memory or working memory? Scandinavian Audiology, 28, 241-247.

Baayen, R. H., Piepenbrock, R., \& Gulikers, L. (1995). The Celex lexical database (Release 2) [CD-ROM]. Linguistic Data Consortium, University of Pennsylvania, Philadelphia.

Baskill, J., \& Coles, R. (1999, September). Relationship between tinnitus loudness and severity. In J. Hazell (Ed.), Proceedings of the Sixth International Tinnitus Seminar (pp. 424-428). Cambridge, U.K.: The Tinnitus and Hyperacusis Centre.

Broadbent, D. E., Broadbent, M. H. P., \& Jones, J. L. (1986). Performance correlates of self reported cognitive failure and obsessionality. British Journal of Clinical Psychology, 28, 285-299.

Broadbent, D., Cooper, P. F., Fitzgerald, P., \& Parkes, K. R. (1982). The Cognitive Failures Questionnaire and its correlates. British Journal of Clinical Psychology, $21,1-16$.

Crawford, J. R., Parker, D. M., Stewart, L. E., Besson, J. A. O., \& Delacey, G. (1989). Prediction of WAIS IQ with National Adult Reading Test: Cross validation and extension. British Journal of Clinical Psychology, 28, 267-273

Daneman, M., \& Carpenter, P. A. (1980). Individual differences in working memory and reading. Journal of Verbal Learning and Verbal Behaviour, 19, 450-466.

Davis, A., \& Roberts, H. (1996). Tinnitus and health status: SF-36 profile and accident prevalence. In G. Reich \& J. Vernon (Eds.), Proceedings of the 5th International Tinnitus Seminar (pp. 257-265). Portland, OR: American Tinnitus Association.

Eysenck, M. W., \& Calvo, M. G. (1992). Anxiety and performance: The processing efficiency theory. Cognition and Emotion, 6, 409-434.

Folmer, R., Griest, S., \& Martin, W. (2001). Chronic tinnitus as phantom auditory pain. Archives of Otolaryngology-Head \& Neck Surgery, 124, 394-400.
Gatehouse, S. (1991). The role of non-auditory factors in measured and self report disability. Acta OtoLaryngologica (Suppl. 476), 249-256.

Granick, S., Kleban, M. H., \& Weiss, A. D. (1976). Relationships between hearing loss and cognition in normally aged persons. Journal of Gerontology, 31, 434-440.

Halford, J. B., \& Anderson, S. D. (1991). Anxiety and depression in tinnitus sufferers. Journal of Psychosomatic Research, 35, 383-390.

Hallam, R. S. (1986). Psychological approaches to the management of tinnitus distress. In J. W. P. Hazell (Ed.), Tinnitus. London: Churchill Livingstone.

Hallam, R. S., Jakes, S. C., \& Hinchcliffe, R. (1988). Cognitive variables in tinnitus annoyance. British Journal of Clinical Psychology, 27, 213-222.

Hallam, R. S., McKenna, L., \& Shurlock, L. (2004). Tinnitus impairs cognitive efficiency. International Journal of Audiology, 43, 218-226.

Hallam, R., Rachman, S., \& Hinchcliffe, R. (1984). Psychological aspects of tinnitus. In S. Rachman (Ed.), Contributions to medical psychology (Vol. 3, pp. 34-53). Oxford, England: Pergamon Press.

Harris, J., \& Wilkins, A. J. (1982). Remembering to do things: A theoretical framework and illustrative experiment. Human Learning, 1, 1-14.

Hébert, S., Paiement, P., \& Lupien, S. J. (2004). A physiological correlate for the intolerance to both internal and external sounds. Hearing Research, 190, 1-9.

Holgers, K., Erlandsson, S., \& Barrenas, M. (2000). Predictive factors in the severity of tinnitus. Audiology, 39 , 284-291.

Jacobson, G. P., Calder, J. A., Newman, C. W., Peterson, E. L., Wharton, J. A., \& Ahmad, B. K. (1996). Electrophysiological indices of selective auditory attention in subjects with and without tinnitus. Hearing Research, 97, 66-74.

Jakes, S. C., Hallam, R. C., Chambers, C., \& Hinchcliffe, R. (1985). A factor analytical study of tinnitus complaint behavior. Audiology, 24, 195-206.

Jastreboff, P. J., \& Hazell, J. (1993). A neurophysiological approach to tinnitus: Clinical implications. British Journal of Audiology, 27, 7-17.

Johnston, W. A., \& Heinz, S. P. (1978). Flexibility and capacity demands of attention. Journal of Experimental Psychology: General, 107, 420-435.

Johnstone, M., \& Walker, M. (1996). Suicide in the elderly: Recognising the signs. General Hospital Psychiatry, 18, $257-260$.

LaBerge, D. (1975). Acquisition of automatic processing in perceptual and association learning. In P. M. A. Rabbit \& S Dornic (Eds.), Attention and performance, $V$ (pp. 50-64). London: Academic Press.

LaBerge, D. (1990). Attention. Psychological Science, 1, 156-162.

Martin, M., \& Jones, G. V. (1983). Distribution of attention in cognitive failures. Human Learning, 2, 221-226.

McKenna, L., Hallam, R. S., \& Hinchcliffe, R. (1991). The prevalence of psychological disturbance in neuro-otology 
outpatients. Journal of Clinical Otolaryngology, 16, 452-456.

McKenna, L. M., Hallam, R. S., \& Shurlock, L. (1996). Cognitive functioning in tinnitus patients. In G. Reich \& J. Vernon (Eds.), Proceedings of the 5th International Tinnitus Seminar (pp. 589-595). Portland, OR: American Tinnitus Association.

Murphy, D. R., \& Wilcox, K. A. (1999). Inhibition and aging: Similarities between younger and older adults as revealed by the processing of unattended auditory information. Psychology and Aging, 14, 44-59.

Neisser, U., \& Becklen, P. (1975). Selective looking: Attending to visually superimposed events. Cognitive Psychology, 7, 480-494.

Nelson, H. (1991). The National Adult Reading Test (2nd ed.). Windsor, U.K.: NFER-Nelson.

Newman, C. W., Wharton, J. A., \& Jacobson, G. P. (1997). Self-focus and somatic attention in patients with tinnitus. Journal of American Academy of Audiology, 8, 143-149.

Pearman, A., Freidman, L., Brooks, J. O., \& Yesavage, J. A. (2000). Hearing impairment and serial word recall in older adults. Experimental Aging Research, 26, 383-391.

Posner, M., \& Snyder, C. (1975). Facilitation and inhibition in the processing of signals. In P. M. A. Rabbit \& S. Dornic (Eds.), Attention and performance, V (pp. 669-682). New York: Academic Press.

Reason, J. (1988). Stress and cognitive failures. In S. Fisher \& J. Reason (Eds.), Handbook of life stress, cognition, and health (pp. 405-421). New York: Wiley.

Rosch, E., \& Mervis, C. B. (1975). Family resemblances: Studies in the internal structure of categories. Cognitive Psychology, 7, 573-605.

Rutter, D. R., \& Stein, M. J. (1999). Psychological aspects of tinnitus: A comparison with hearing loss and ear, nose and throat disorders. Psychology of Health, 14, 711-718.

Schneider, B. A., Daneman, M., \& Pichora-Fuller, M. K. (2002). Listening in aging adults: From discourse comprehension to psychoacoustics. Canadian Journal of Experimental Psychology, 56, 139-152.

Schneider, W., \& Shiffrin, R. (1977). Controlled and automatic human information processing: I. Detection, search and attention. Psychological Review, 84, 1-66.

Shiffrin, R. M., \& Schneider, W. (1977). Controlled and automatic human information processing: II. Perceptual learning, automatic attending and a general theory. Psychological Review, 84, 127-190.

Sindhusake, D., Mitchell, P., Golding, M., Newall, P., Rochtchina, E., \& Rubin, G. (2003). Prevalence and characteristics of tinnitus in older adults. The Blue Mountains hearing study. International Journal of Audiology, 42, 289-294.

Smith, E. E., \& Medin, D. L. (1981). Categories and concepts. Cambridge, MA: Harvard University Press.

Spielberger, C. D., Gorsuch, R. L., Lushene, R. E., Vagg, P., \& Jacobs, G. A. (1983). Manual for State Trait Anxiety Inventory. Palo Alto, CA: Consulting Psychologist Press.

Stevens, C., Walker, G., \& Gallagher, M. (2005). Severe tinnitus and its effect on selective and divided attention. 32nd Australasian Experimental Psychology conference [Abstract]. Australian Journal of Psychology, 57(Suppl).

Stroop, J. R. (1935). Studies of interference in serial verbal reactions. Journal of Experimental Psychology, 18, 643-662.

Turner, M. L., \& Engle, R. W. (1989). Is working memory capacity task dependent? Journal of Memory and Language, 28, 127-154.

Tyler, R. S., \& Baker, L. J. (1983). Difficulties experienced by tinnitus sufferers. Journal of Speech and Hearing Disorders, 48, 150-154.

Vesterager, V. (1997). Fortnightly review: Tinnitus investigation and management. British Medical Journal, 314, 728-731.

Walpurger, V., Hebing-Lennartz, G., Denecke, H., \& Pietrowsky, R. (2003). Habituation deficit in auditory event-related potentials in tinnitus complainers. Hearing Research, 181, 57-64.

Wechsler, D. (1955). Manual for the Wechsler Adult Intelligence Scale. New York: The Psychological Corporation.

Wilson, P. H., Henry, J., Bowen, M., \& Haralambous, G. (1991). The Tinnitus Reaction Questionnaire: Psychometric properties of a measure of distress associated with tinnitus. Journal of Speech and Hearing Research, 34, 197-201.

Received January 14, 2004

Accepted July 3, 2005

DOI: 10.1044/1092-4388(2006/012)

Contact author: Catherine Stevens, School of Psychology/ MARCS, University of Western Sydney-Bankstown, Locked Bag 1797, South Penrith, DC NSW 1797, Australia. E-mail: kj.stevens@uws.edu.au 